\title{
Calibration of fibre-optic RF E/H-field probes using a magnetic reso- nance (MR) compatible TEM cell and dedicated MR measurement techniques
}

\author{
T. Klepsch ${ }^{1}$, T. D. Lindel ${ }^{2}$, W. Hoffmann ${ }^{2}$, H. Botterweck ${ }^{3}$, B. Ittermann ${ }^{2}$, F. Seifert ${ }^{2}$ \\ ${ }^{1}$ Medical Sensors and Devices Laboratory, Luebeck University of Applied Sciences, Luebeck, Germany \\ ${ }^{2}$ Physikalisch-Technische Bundesanstalt (PTB), Berlin, Germany \\ ${ }^{3}$ Laboratory of Medical Imaging, Luebeck University of Applied Sciences, Luebeck, Germany \\ E-mail: tobias.klepsch@fh-luebeck.de
}

\begin{abstract}
Supervision of specific absorption rate (SAR) during Magnetic Resonance Imaging (MRI) is crucial for patient safety. Electromagnetic field (EMF) simulations have proven to be essential tools in design and evaluation of dedicated MR coil setups. However, such simulations can only be validated comprehensively by physical measurements of EM field distribution using radio frequency (RF) electric and magnetic field probes. The reliability of those measurements strongly depends on the quality of the calibration procedure. This work presents a fully MR compatible Transverse Electromagnetic Cell (TEM cell) which was used to calibrate two different fibre coupled field probes by common MR measurement techniques only. In a water filled capillary mounted inside the TEM cell the $\mathrm{B}_{1}{ }^{(+)}$magnetic field component produced by the cell was measured by a flip angle mapping sequence. In a TEM cell the electric field and the magnetic field are related via $\mathrm{E} / \mathrm{H}=376.73 \Omega$. Therefore, the E-field can be related exactly to $\mathrm{B}_{1}{ }^{(+)}$by $\mathrm{E}=2 \mathrm{c}_{0} \mathrm{~B}_{1}{ }^{(+)}$( $\mathrm{c}_{0}$ : velocity of light). For the acquisition of the sensor signals the receiver unit of a clinical 3T MR scanner and a 7T whole-body research scanner was used; as a result, no additional hardware was necessary. The estimated accuracy of the calibration procedure is $5 \%$ or $0.4 \mathrm{~dB}$ and the linear behaviour of the calibrated sensor signals was verified. For further evaluation of uncertainties and homogeneity of the generated RF fields an improved design of the TEM cell with a characteristic impedance of $50 \Omega$ is desired.
\end{abstract}

\section{Introduction}

Supervision of SAR in high and ultrahigh field MRI is an inevitable requirement for patient safety [1]. EMF simulations of RF coils and inner body field distributions have proven to be essential tools to estimate the possible energy deposition in MR examinations [2]. However, a complete experimental validation of those simulations needs measurements of both E- and H-field components by appropriate MR compatible RF field probes [3]. The reliability of such measurements strongly depends on the quality of the probe's calibration. This work presents a fully MR compatible TEM cell, which can be used within clinical MR scanners to calibrate fibre-optic field probes. For signal reception the multichannel receiver unit of the MR scanner is used to acquire the sensor signals, hence, no additional hardware is needed.

TEM cells were introduced in the 1970s by Crawford [4]. They are widely used in the traceable generation of RF fields and for EM compatibility testing of electronic devices [5]. In a TEM cell the electric field is related exactly to the magnetic field by $\mathrm{E} / \mathrm{H}=376.73 \Omega$ [6]. The $\mathrm{B}_{1}$-field generated by an RF coil can be decomposed into two circularly polarized components $\mathrm{B}_{1}{ }^{(+)}$and $\mathrm{B}_{1}{ }^{(-)}$where the first rotates along with the nuclear spins of the water protons [7]. Therefore, a calibration of the electric field of the
TEM cell can be performed by a measurement of $\mathrm{B}_{1}^{(+)}$ leading to the relation:

$$
\mathrm{E}=2 \mathrm{c}_{0} \mathrm{~B}_{1}^{(+)}
$$

$\left(\mathrm{c}_{0}\right.$ : velocity of light).

\section{Methods}

This section presents the methods according to the used TEM cell and the EM field probes as well as the calibration procedures at the MR scanners.

\subsection{TEM cell}

The TEM cell is an open double stripline design taken from available FDTD (Finite Differences in the Time Domain Method) EM simulation data. The conductors consist of standard FR4 circuit boards ( $18 \mu \mathrm{m}$ copper layer) to minimize eddy current artifacts. The distance between the layers of the stripline is $3 \mathrm{~cm}$, enabling the mounting of a probe head inside the cell. The termination of the cell on both sides consists of two tapered feeding sections stabilized with two copper sheets $(1 \mathrm{~cm} \mathrm{x} 1 \mathrm{~cm})$. The geometry of the cell was chosen to result in a characteristic impedance of $50 \Omega$. Semi rigid cables equipped with nonmagnetic SMA connectors form the feeding ports of the 
cell. As shown in Figure 1, the upper of the two symmetric measurement cavities contains a water filled capillary made of Perspex (inner diameter: $3 \mathrm{~mm}$, outer diameter: $5 \mathrm{~mm}$ ) to enable measurements of $\mathrm{B}_{1}{ }^{(+)}$using a preparation pulse sequence [1].

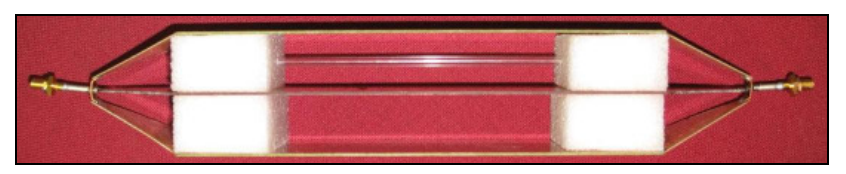

Figure 1 TEM cell with water filled capillary

\subsection{Fibre-optic field sensors}

The time-domain RF E-field sensor (OEFS-S1B, Seikoh Giken, Japan) uses a fully electro-optic (OEFS) design and consists of three sensor channels equipped with three Mach-Zehnder type electro-optic E-field sensor chips [8]. However, this work only used one channel. By rotation of the sensor head in steps of $120^{\circ}$, all three complex valued E-field components were measured. The probe head is coupled to a controller unit via $10 \mathrm{~m}$ of optical fibres.

The time-domain H-field sensor (H-TDS, SPEAG Inc., Switzerland) consists of a probe head, optical fibres for signal transmission, and a controller unit [9]. A tiny pickup-loop is embedded in the probe head. A power laser provides the probe head with electrical power while the picked up RF signal is converted into an optical signal via a vertical surface emitting laser (VCSEL). A second optical fibre is used for subsequent transmission of the RF signal to the controller unit of the sensor.

\subsection{Calibration procedure}

For the calibration of both RF field probes, the TEM cell was operated inside two different whole-body MR scanners with magnetic flux densities of $3 \mathrm{~T}$ (Magnetom Verio, Siemens-Healthcare, Erlangen, Germany) and $7 \mathrm{~T}$ (Magnetom, Siemens-Healthcare, Erlangen, Germany). A $50 \Omega$ dummy load was connected to one port of the cell in order to terminate the transmission line. The TEM cell was installed on the patient table of the MR scanner. The controller units for both probes were placed outside the RF shield of the scanning room to avoid EM interferences [10].

\subsubsection{Calibration of the OEFS}

By an adjustable gantry, the OEFS was placed in the centre of the TEM cell. The principal axis of the cell was adjusted perpendicular to the $\mathrm{z}$-axis of the scanner (Figure 2). The RF output of the sensor was connected to one channel of the receiver unit of the scanner. The electric field was measured using rectangular RF pulses with a pulse width of $5.12 \mathrm{~ms}$ and five different nominal transmitter voltages $\left(\mathrm{U}_{\mathrm{TX}}=0,30,60,90,120,150 \mathrm{~V}\right)$ at all three angular positions of the OEFS. $\mathrm{B}_{1}^{(+)}$-maps were acquired in a central sagittal slab (thickness $20 \mathrm{~mm}$ ) of the water capillary.
In the 7T MR scanner, the TEM cell was placed with its long axis parallel to the $\mathrm{z}$-axis of the scanner due to the reduced diameter of the magnet bore. Because of reduced field homogeneity in the long axis, the water capillary was placed perpendicular to the scanner's z-axis. Accordingly, the same acquisition procedures for the $\mathrm{B}_{1}{ }^{(+)}$-maps and the OEFS sensor signals as in the $3 \mathrm{~T}$ measurements could be applied.

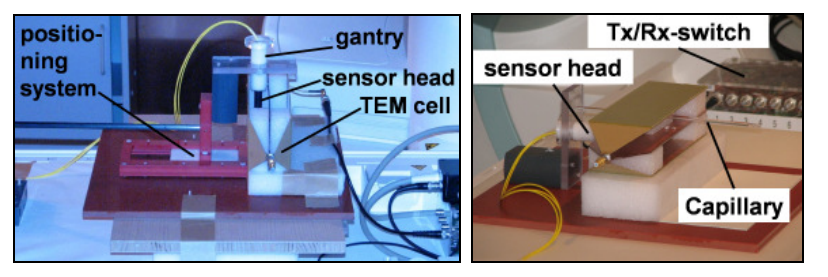

Figure 2 OEFS in the $3 \mathrm{~T}$ scanner (left) and at $7 \mathrm{~T}$ (right)

\subsubsection{Calibration of the H-TDS}

For the calibration of the H-TDS, the TEM cell was adjusted in parallel orientation to the $\mathrm{z}$-axis of the MR scanner (Figure 3). The pick-up-loop within the probe head of the H-TDS is oriented in parallel to the probe's axis. Therefore, the probe head had to be placed inside the measurement cavity of the TEM cell under an angle of $45^{\circ}$ with respect to the axis of the cell to get a sufficient signal. After a first measurement, the probe head was rotated by $90^{\circ}$ and the magnetic field was measured again allowing measurement of the vector sum of both signals and reduction of uncertainties due to unavoidable tilting of the cell. In addition, the measurements were repeated for a sensor mounting position of $135^{\circ}$. At $7 \mathrm{~T}$, the water capillary was again placed perpendicular to the principal axis of the TEM cell.
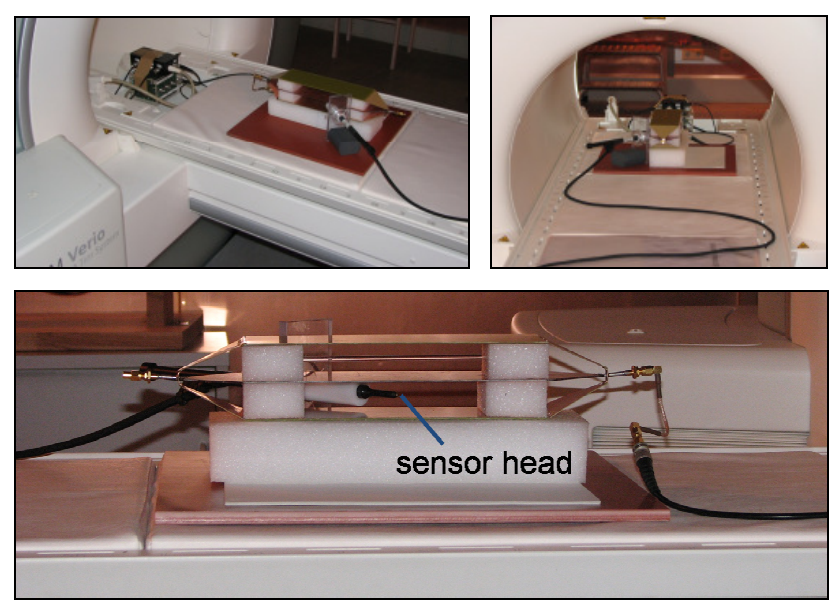

Figure 3 H-TDS and TEM cell on the patient table of the 3T scanner

\section{Results}

Figure 4 shows the time course of the output of the OEFS for a rectangular transmitter pulse of $5.12 \mathrm{~ms}$ and amplitude of $100 \mathrm{~V}$. For different transmitter voltages (adjusted 
on the scanner console) the linearity of the sensor signal can be observed in Figure 5. At $123.3 \mathrm{MHz}$ and a transmitter voltage of $100 \mathrm{~V}$, a value of $\left|\mathrm{B}_{1}{ }^{(+)}\right|=(10.0 \pm 0.3) \mu \mathrm{T}$ was obtained. From Eq. (1) the corresponding E-field was calculated to be $(3104 \pm 155) \mathrm{V} / \mathrm{m}$ which resulted in a sensor signal of 0.00397 in scanner specific units. For the $7 \mathrm{~T}$ measurements at a frequency of $297 \mathrm{MHz}$, a comparable linearity of the sensor output signal was verified (Figure 6).

The H-TDS saturated at $3 \mathrm{~T}$ for voltages above $130 \mathrm{~V}$ as seen in Figure 7. Therefore, at $7 \mathrm{~T}$ the calibration was performed for a transmitter voltage of $20 \mathrm{~V}$ and a magnetic field of $\left|\mathrm{B}_{1}^{(+)}\right|=(2.4 \pm 0.1) \mu \mathrm{T}$ was obtained.

The characteristic impedance of the TEM cell was measured to be $53 \Omega$ in agreement with FDTD simulations. Taking into account this systematic mismatch between the calculated electrical field and the measured field we estimated the uncertainty of the calibration to be about $5 \%$.

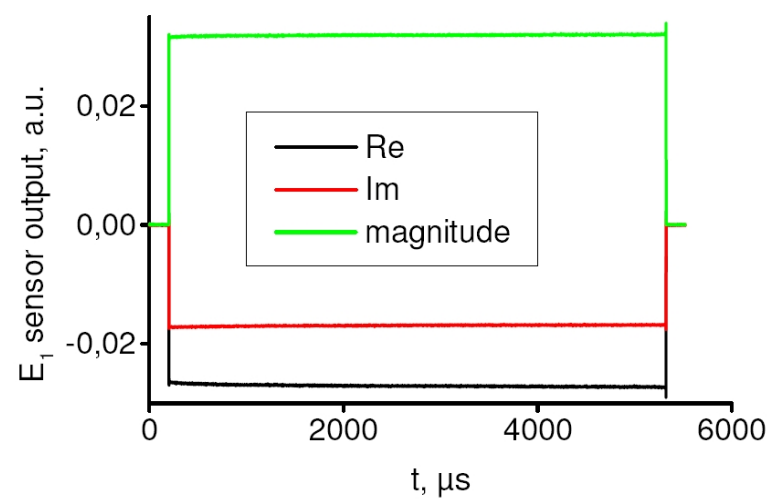

Figure 4 Sensor output of the OEFS for a rectangular transmitter pulse (sum of 10 scans) [10]

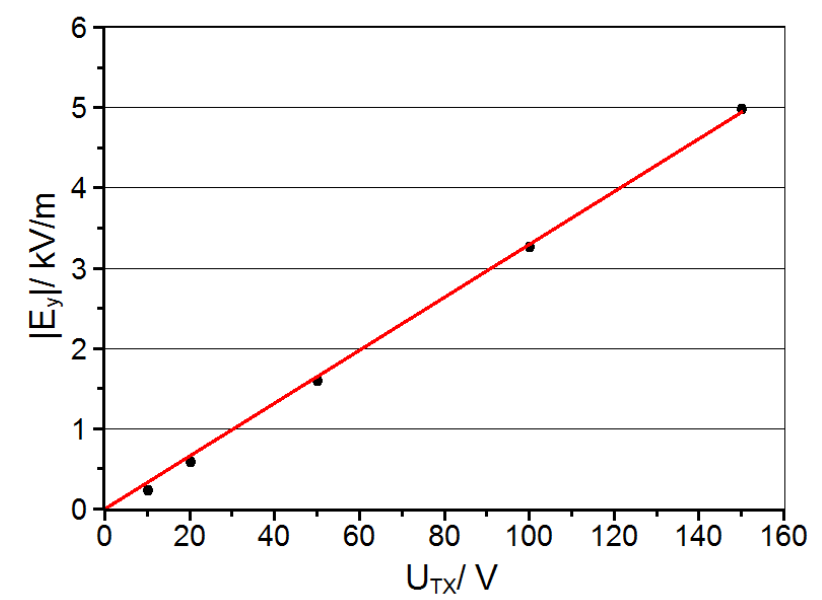

Figure 5 Calibrated sensor output signal of the OEFS for different transmitter voltages at $3 \mathrm{~T}$

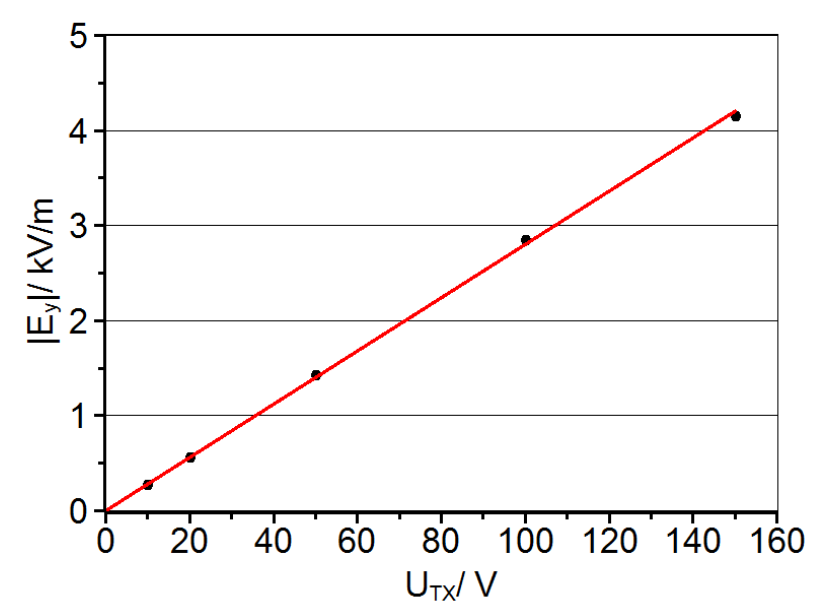

Figure 6 Calibrated sensor output signal of the OEFS for different transmitter voltages at $7 \mathrm{~T}$

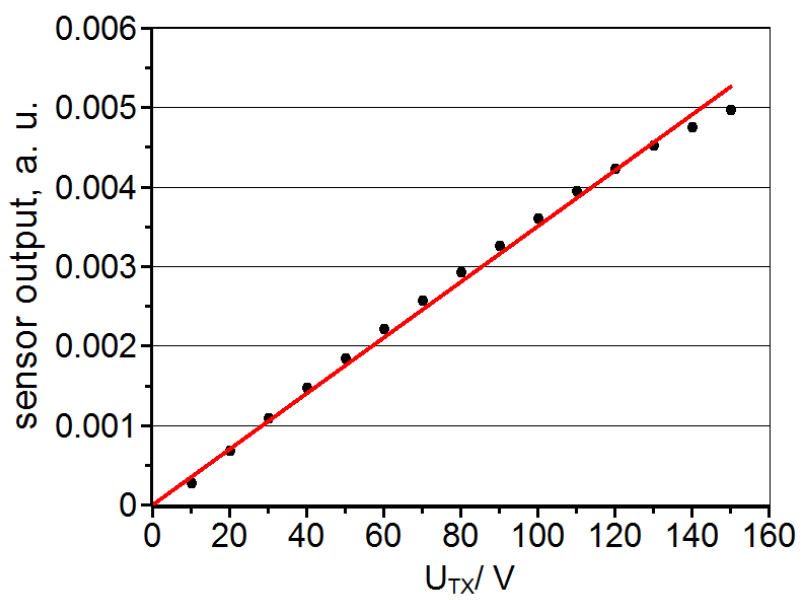

Figure 7 Sensor output signal of the H-TDS for different transmitter voltages at $3 \mathrm{~T}$

\section{Conclusion}

We have introduced a fully MR compatible TEM cell that is used for the calibration of time-domain electro-optic EM field sensors. The estimated uncertainty of the calibration is $5 \%$ or $0.4 \mathrm{~dB}$. Due to the absolute calibration via measurements of the $\mathrm{B}_{1}{ }^{(+)}$field component generated by the cell, no further correction regarding feeding lines is needed. Because of the broadband nature of the TEM cell, the calibration procedure can be utilized in any clinical MR system. This was demonstrated by measurements in a 3T and a 7T MR scanner at $123 \mathrm{MHz}$ and $297 \mathrm{MHz}$, respectively. According to our knowledge, this is the first calibration of an electro-optic field sensor in a clinical scanner using the nuclear spin of the water proton as an intrinsic field probe.

The actual characteristic impedance of the prototype TEM cell of $53 \Omega$ resulted in a systematic mismatch between the calculated electrical field and the measured field. A redesigned geometry creating a TEM cell with a characteristic 
impedance of $50 \Omega$ is intended to further improve accuracy.

The application of the calibrated EM probes is intended for the evaluation of RF field distributions of MR coils and the validation of corresponding FDTD EM field simulations. Exemplarily, the quantitative agreement of FDTD EM simulation profiles and calibrated EM sensor measurements for two different simple coil geometries at $3 \mathrm{~T}$ and $7 \mathrm{~T}$ could be shown and thus a reliable calibration procedure is to be expected [11].

\section{Acknowledgements}

This work was supported by EMRP grant HLT06. The EMRP is jointly funded by the EMRP participating countries within EURAMET and the European Union.

\section{References}

[1] Seifert F., Wuebbeler G., Junge S., Ittermann B. and Rinneberg H.: Patient safety concept for multichannel transmit coils, JMRI 26 (2007) 1315-1321

[2] Collins C. M., Wang Z.: Calculations of radio frequency electromagnetic fields and their effects in MRI of human subjects, MRM 65 (2011) 1470-1482

[3] Seifert F., Lindel T. D. and Ullmann P.: Evaluation of E-field distributions in parallel transmit systems by time-domain optical electric-field sensors, Proc. Intl. Soc. Mag. Reson. Med. 18 (2010) 1452

[4] Crawford M. L.: Generation of standard fields using TEM transmission cells, IEEE Trans. EMC, Vol. EMC-16, No. 4, Nov. 1974

[5] Münter K., Spitzer M. and Glimm J.: Rückführbare Erzeugung hochfrequenter elektromagnetischer Felder, Technisches Messen: 69 (2002), 2, 65-69

[6] Schwab A. J.: Elektromagnetische Verträglichkeit, 4. neu bearbeitete Auflage, Springer-Verlag, Berlin, 1996

[7] Hoult D. I.: The principle of reciprocity in signal strength calculations - a mathematical guide, Concepts Magn Reson 2000, 12: 173-187

[8] Seikoh Giken Co. Ltd.: Isotropic 3-axis Optical EField Sensor OEFS-S1B, Technical Manual, 2008

[9] Schmidt und Partner Engineering AG, HTDS Operating Manual, Switzerland, 2010

[10] Seifert F., Klepsch T., Lindel T. D., Hoffmann W., and Ittermann B.: TEM cell for calibration of an electro-optic E-field sensor in a clinical scanner, Proc. Intl. Soc. Mag. Reson. Med. 19 (2011) 3777

[11] Klepsch T.: Aufbau und Charakterisierung einer MR-kompatiblen TEM-Zelle zur Kalibration elektromagnetischer Feldsensoren mit MR-Messtechniken, Diploma Thesis, Luebeck University of Applied Sciences, Luebeck, 2011 